\title{
Landscape-scale spatial planning at WWF: a variety of approaches
}

\author{
John Morrison, Colby Loucks, Barney Long and Eric Wikramanayake
}

\begin{abstract}
WWF's spatial landscape planning methods are diverse, reflecting WWF's global, decentralized organizational structure. Over the past decade WWF's spatial planning methods have varied from expert-only workshops to systematic conservation planning using decision support software, and combinations of both. We provide four case studies from the Asia-Pacific region to illustrate the variety of approaches that have been used, emphasizing assessment directed at implementation. The method appropriate to each situation was chosen based on data availability, timing, costs, available range of stakeholders, and the technical facility and interest of the stakeholders themselves. In all cases, methods were chosen to balance staff technical capacity, technical rigour, and political buy-in, hoping to ensure that the resulting plan would actually be implemented.
\end{abstract}

Keywords Ecoregions, expert workshops, landscapes, systematic conservation planning, WWF

\section{Introduction}

7 he World Wildlife Fund's (WWF) approach to largescale conservation planning began in the late 1990s with the development of the Global 200, a biodiversity prioritysetting exercise that used a representative approach to select 238 of the earth's most outstanding terrestrial, freshwater and marine systems (Olson \& Dinerstein, 1998). Subsequently, WWF embarked on ecoregion-based conservation planning in which spatial priorities were identified in many of the Global 200 regions. Ecoregions are defined by WWF to be relatively large units of land containing a distinct assemblage of natural communities and species, with boundaries that approximate the original extent of natural communities prior to major land-use change (Olson et al., 2001). Ecoregions are often larger than landscapes but not always. In essence, WWF realized that only at relatively large scales (generally greater than single protected areas) could conservation planning and implementation adequately understand and preserve habitats and ecological processes.

Since the first ecoregion-based conservation planning workshop was held in 1997, for the Chihuahuan Desert, there have been more than 30 ecoregion (or ecoregion complex) analyses. Across all these planning analyses priority areas for

JOHN MORRISON (Corresponding author), COLBY LOUCKs, BARNEY LONG and Eric Wikramanayake WWF, 1250 24th Street, NW, Washington, DC 20037, USA. E-mail john.morrison@wwfus.org

Received 30 July 2008. Revision requested 7 November 2008.

Accepted 13 December 2008. conservation implementation were identified based predominantly on biological characteristics. Priority areas within ecoregions (or ecoregion complexes), while varying greatly in size depending on the specific region and set of local circumstances, form the basis for WWF's landscapescale conservation planning and implementation. However, in some cases, landscape-scale conservation planning has been performed for entire small ecoregions. The same largescale planning concerns (representation of habitats and species in protected areas, incorporation of ecological processes in spatial plans) are believed to be applicable at both ecoregional and landscape scales. However, only at the small-ecoregion and landscape scales does planning address the spatially explicit decisions required to make a conservation plan practically implementable.

There are two important aspects of WWF's approach to spatial landscape planning. Firstly, strategic planning for conservation action is a critical complement to spatial planning. WWF uses a best practice strategic planning framework called the WWF Program Standards (WWF, 2005), which is similar to The Nature Conservancy's Conservation Action Planning and is derived from the Conservation Measures Partnership's Open Standards for the Practice of Conservation (Conservation Measures Partnership, 2007). Ideally, spatial planning and strategic planning are seamlessly integrated.

Secondly, WWF is a network of semi-independent country organizations (often referred to as the WWF Network), and there are no mandated approaches to conservation planning at the ecoregion or landscape scale. Consequently, there is a wide variety of approaches used in different countries and under different situations that have to do with data availability, conservation targets, and opportunities to coordinate with partners. Furthermore, WWF's approach to ecoregion and landscape-scale conservation planning has continued to evolve, especially as systematic conservation planning software and other tools have become increasingly available and user-friendly over the past decade.

WWF's landscape level conservation planning approach aims to address the goals of: (1) Representing all distinct natural communities within conservation landscapes and protected area networks. (2) Maintaining ecological and evolutionary processes that create and sustain biodiversity. (3) Maintaining viable populations of species. (4) Conserving blocks of natural habitat that are large enough to be resilient to large-scale stochastic and deterministic disturbances as well as to long-term changes (Dinerstein et al., 200ob, modified from Noss, 1992). 
Our aim here is to describe examples of the range of landscape-scale planning approaches that have typically been used by the WWF Network, with particular focus on how and why decisions were made during the assessment process but also looking forward to implementation. We have chosen four case studies from the Asia-Pacific region to illustrate the diversity of expert and stakeholder input and the use of systematic conservation planning principles.

\section{Methods}

Knight et al. (2006a,b) divided conservation planning into conservation assessment and implementation. This paper focuses on conservation assessment but the process supporting the assessment is also considered as it relates to implementation. Conservation assessment methods used in the WWF Network vary considerably, even within a single region such as Asia-Pacific. Some are strictly stakeholderbased workshops whereas others involve planning software (with or without stakeholders). We describe four types of assessment processes used across the WWF Network.

\section{Stakeholder-based, hand-drawn spatial planning workshop}

This method is based on multi-day workshops at which experts are distributed first into taxonomic, then subregional groups, where they discuss and identify priority areas for the conservation of ecosystems, communities, species assemblages, species, and subspecies. Large-format printed maps are provided and the experts develop consensus priority areas, which are drawn directly on the maps. These polygons are digitized and presented for group discussion. Eventually the polygons from the taxonomic and sub-regional groups are integrated in a group process. In poorly studied landscapes, pre-workshop desk studies are commissioned to assemble the information for the region.

Ecosystems, natural communities and species integrated into the priority areas are described in data sheets that accompany each hand-drawn priority area. The areas are intended to cover all native habitats, and keystone and threatened species, and ecological processes required to maintain them.

The priority areas are identified and ranked based on endemism, unique and/or intact habitats, and unique or extant ecological processes. An emphasis is placed on identifying areas that represent all native habitats and species. The boundaries and spatial extent of the priority areas are based on the knowledge and expertise of the participants. Multi-use corridors between priority areas are also identified, and areas are ranked for biological importance and threat status. Quantitative objectives may or may not be specified, although the extent and location of the priority areas can be quantified after they are identified. Because costs are not incorporated into this method the final maps are not the most efficient solution. This process can be completed in as few as 3 months, although at least 6 months are preferable. Descriptions and examples of this type of spatial planning can be found in Mittermeier et al. (1994), Dinerstein et al. (2000a), Baltzer et al. (2001), Kramer \& Kramer (2002) and Wikramanayake et al. (2001a).

One-time stakeholder workshop, with use of planning software

Another method that relies on a multi-day workshop to gather the input of stakeholders makes use of conservation planning software. The basic tenet is to develop a number of planning scenarios using systematic conservation planning software before the workshop (Ball \& Possingham, 2000), in which options are presented to local, regional and expert stakeholders. Adjustments are made to the assessment, as necessary, during the workshop.

The scenarios are developed using the process described by Margules \& Pressey (2000). The key conservation elements are chosen by a small planning team in advance of the workshop, sometimes during $>_{1}$ year of consideration and outside consultation. Priorities generally focus on key habitats and species (the latter if species data are available). Fine-scale special elements are also selected as targets (geomorphological, hydrological or species-related, mappable features such as caves, beach ridges or other restricted and/or keystone habitats; Noss, 1990; Pressey, 2004; Pressey et al., 2007). Four to six scenarios are run, with each scenario a different set of representation goals (e.g. 20, 30 and 40\% representation). Typically, unchanging and high (c. 100\%) goals are set for fine-scale special elements. The planning software allows the use of cost layers to prioritize the suite of priority areas. Cost layers may be threat-based or actual cost-based (either cost to purchase or cost to manage) and the planning software chooses an efficient portfolio of sites at the least cost.

If possible, the participants are asked to make preliminary decisions on representation goals before they have seen the maps of the scenarios. The options and implications of the various representation levels are presented to the group, and a consensus is reached before any scenario maps are presented. Then the maps are distributed to subregional groups for review. The participants then evaluate their decisions, and make minor modifications and configuration adjustments, accounting for various government plans as well as conservation biology considerations. As in the previous method, in poorly studied areas pre-workshop studies may be commissioned to assemble available information. This type of assessment requires 1-2 years. This process has been used in New Guinea and Madagascar but is as yet unpublished. 


\section{Planning software-driven process}

A number of WWF regions have used a traditional planning software-driven approach, with varying stakeholder involvement. Such processes are also closely aligned with the process described in Margules \& Pressey (2000). Combinations of ecosystems, natural communities, species assemblages and species are typically selected by a small planning team or with stakeholder input, to provide habitat and species representation and to capture unique special elements and ecological processes (Noss, 1990; Pressey, 2004; Pressey et al., 2007). Cost layers are also used in this method. To date, WWF has used threats (e.g. roads or developed areas) as the primary cost layers. Planning units are also required, and they have been computer-generated pixels, hexagons, small watersheds or, in some cases, small administrative units. The time for plan development is variable but generally $1-3$ years.

\section{Spatial planning for ecological processes}

In some situations programmes have carried out spatial planning solely on the basis of a key ecological process. In this case habitat representation or representation of special elements are not comprehensively considered. The ecological process is typically connectivity, either terrestrial or hydrological, and often predicated on the spatial requirements of large mammals. Various computing techniques are used, including hydrological software for freshwater environments and least-cost path analysis in terrestrial environments, to identify corridors. Such modelling will probably become more prevalent and sophisticated, with the increasing use of niche or bioclimatic models of species distribution responses to climatic changes, such as latitudinal and altitudinal connectivity. Such an assessment can be completed in 6-12 months.

\section{Case studies}

Because of the diversity of approaches in the application of spatial conservation assessment in WWF, even within one region, we have included four examples from the AsiaPacific region (Fig. 1). Three of these illustrate comprehensive conservation assessments using expert-based and computer-based systematic conservation planning. We have also included a fourth example that uses large mammal connectivity to develop a conservation assessment.

\section{Vogelkop Montane Forest: expert-based workshop, with government planners}

The region consists of c. $15,000 \mathrm{~km}^{2}$ of tropical montane rainforests encompassing the Arfak and Tamrau mountain ranges in the northern Vogelkop Peninsula of Indonesian
New Guinea, and the immediately surrounding lowland and coastal areas. The montane forest in this ecoregion is dominated by Castanopsis in the lower elevations but with altitude the vegetation changes to moss-draped, Antarctic beech Nothofagus forests, which sometimes occur as monotypic stands, and then to coniferous forests of Podocarpus, Dacrycarpus, Dacridium and Papuacedrus (Petocz, 1989). Although richness and endemism are only moderate compared with some other regions in South-east Asia, the region has $>25$ endemic or near-endemic birds and mammals. This area was identified for further planning by WWF-Indonesia in part because it was included as a Global 200 Ecoregion (Olson \& Dinerstein, 1998).

There are already extensive protected areas in the Vogelkop region, and the planning process aimed to delineate unrepresented habitats for inclusion in protected area extensions, and to integrate conservation thinking with district-level government spatial planning. The process used was the expert stakeholder-based approach, where participants were taken through several days of hand-drawing on large format maps. The Vogelkop planning area is a part of Manokwari and Sorong districts. The planning was promoted to both district governments through integrated planning among government agencies. The participants consisted of university, non-governmental and government staff, as well as local parliamentarians from both districts. The presence at the planning workshop of government stakeholders and recognized experts greatly facilitated the eventual acceptance of the blueprint by some of the relevant government agencies. This process did not directly involve indigenous people although they are present in the planning area.

Because local governmental and civil stakeholders were involved from the start and therefore supportive, the plan has resulted in significant implementation, although it did not occur immediately. Since the original workshop in Manokwari in October 2003, the provincial West Papuan government was restructured into the new provinces of West Papua and Papua. This situation made it difficult to coordinate with both district authorities because they were transitioning to coordination with an entirely new provincial government. The local, district, and provincial governance system needed time to become stable and established. Eventually, the conservation vision was adapted by Sorong district, which used it in their district spatial plan. Implementation is pending in other districts and WWF continues to monitor the process.

\section{TransFly Savannahs: expert-based workshop using systematic conservation planning, with government planners}

This landscape covers the southern reaches of New Guinea, being split between Indonesia to the west and Papua New 


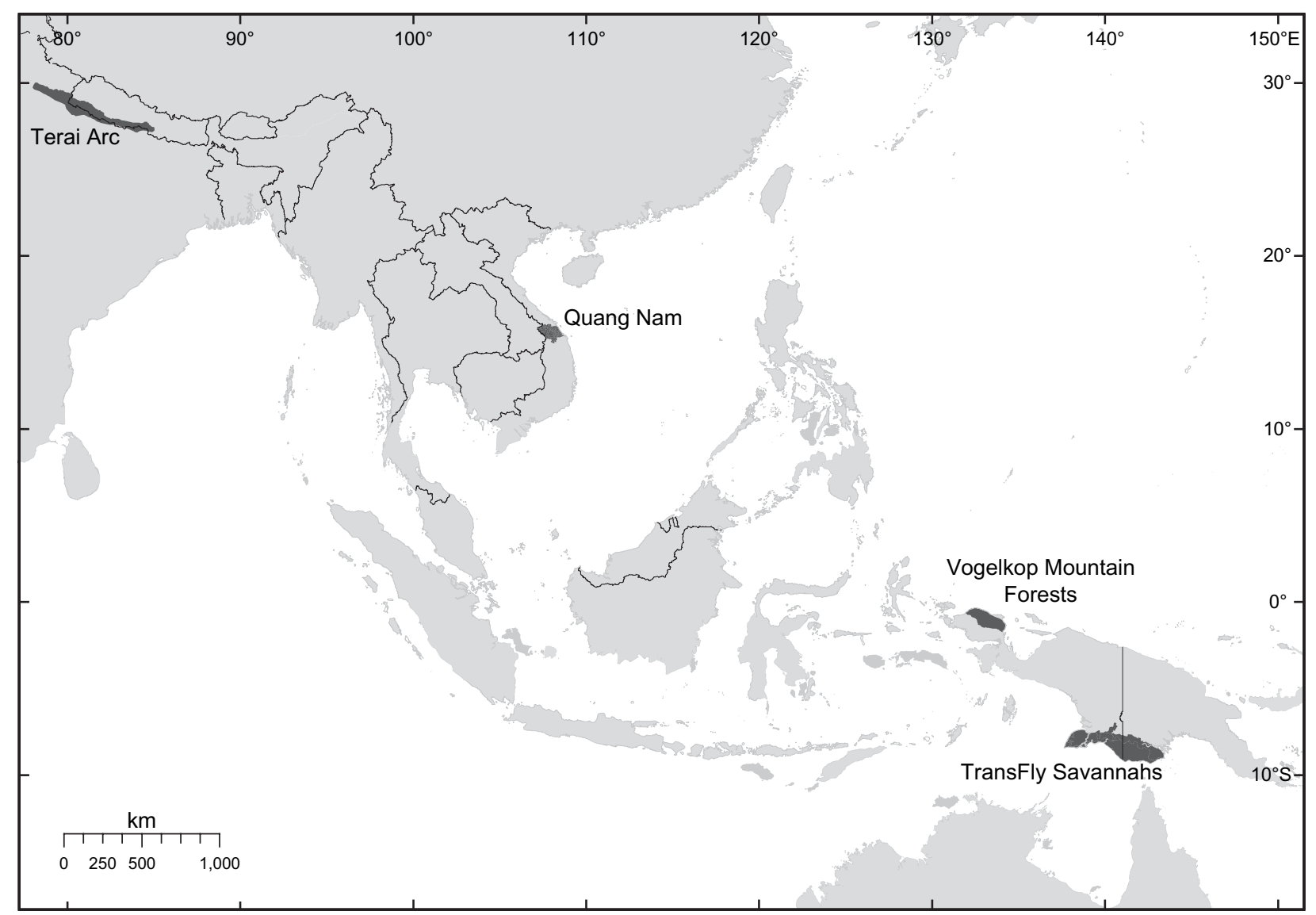

FIG. 1 Locations of the four WWF case studies in Indomalaya and Australasia.

Guinea to the east. It is a vast (c. $\left.100,000 \mathrm{~km}^{2}\right)$ complex of monsoon forest, swamp forests, melaleuca forests, grasslands and mangroves. This complex is a result of extensive lowlands and a seasonal climate, with distinct dry and wet seasons. The area is a very different habitat than the tropical rainforest of most of the island yet integrates many of New Guinea's unique species with Australian elements. Thus, richness is high but endemism is low. This area was highlighted for additional planning by WWF-Indonesia and WWF-South Pacific, in part based on its inclusion in the Global 200 Ecoregions (Olson \& Dinerstein, 1998).

WWF has had a programme associated with the TransFly for $>10$ years. Conservation planning in Melanesia must account for the desires of local people, as land is traditionally owned. While traditional protected areas are present on the Indonesian side, the Papua New Guinea government owns little land and protected areas are generally communitymanaged Wildlife Management Areas. The conservation planning process for the TransFly explicitly incorporated local people to the extent that traditional sacred areas and ancestor routes have been mapped with global positioning systems so that they could be protected according to local wishes.

In some regions it is difficult to bring all relevant stakeholders together more than once. For the TransFly the spatial planning process used a single workshop to select among scenarios generated using planning software (MARXAN; Ball \& Possingham, 200o) before the workshop. A large (c. 70 person) expert-stakeholder workshop was held in May 2006. Because of extreme lack of information for the planning area, the pre-planning phase took $>2$ years. Before planning could commence several desk studies documented what was known about the distribution of birds, mammals and fishes.

Because of the traditional Melanesian land ownership patterns local commitment and support to the process was a key consideration. The area is inhabited by eight sub-tribes of the Malind Anim Ha tribe. Extensive ground mapping of traditional sites, ancestor routes and other areas of cultural importance were mapped in detail with global positioning systems before the workshop.

The presence of government planners, local stakeholders and experts at the workshop produced a robust consensus. This process and result was fully endorsed by the sub-tribes and they signed a map of the conservation vision: a critical gesture of support. The process was also recognized by Merauke district government on the Indonesian side. This made it possible to incorporate the resulting planning maps into ongoing planning efforts in both Indonesia and Papua New Guinea. 
On the Papua New Guinea side habitat representation in Wildlife Management Areas was already significant and mapping for additional protection was ongoing. The Indonesian side has considerably higher population pressure and pressure for development, and thus additional protection on the Indonesian side was a key objective. Since the original workshop in May 2006 the TransFly conservation vision has been integrated into the Merauke District Spatial Plan on the Indonesian side of the border.

\section{Quang Nam Landscape: systematic conservation planning, with government planners}

The Quang Nam landscape is a province in Vietnam's Central Annamite Mountains. It is a portion of a priority landscape identified through a series of ecoregion-based workshops, with expert input for the forests of the Lower Mekong Ecoregion Complex (Baltzer et al., 2001). The Quang Nam landscape falls within the Annamite Range Moist Forests Global 200 Ecoregion (Olson \& Dinerstein, 1998). The region was selected as a priority because of its unique assemblages of birds, butterflies and amphibians and its high priority for mammal conservation (with endemics such as grey-shanked douc Pygathrix cinerea and the saola Pseudoryx nghetinhensis). The diversity of the landscape is threatened by intensive over-harvesting of biodiversity for commercial, often illegal, sale and by fragmentation from infrastructure development.

The Quang Nam Landscape comprises Quang Nam Province, chosen as the unit of study because in Vietnam provinces are responsible for developing their own socioeconomic, development and forestry plans. The objective was to create a spatial prioritization plan institutionalized within the provincial government. This would allow conservation resources to be directed to the areas of highest priority and provincial planning processes to take account of biodiversity priorities when allocating budgets, planning infrastructure development, preparing forest management and harvest plans, and writing socio-economic development plans.

A stakeholder analysis (Long, 2005) showed that provincial level government and their line departments would have most power and influence over both planning and implementation. Therefore, provincial level departments formed the core planning team, led by the Forest Protection Department in consultation with $>40$ provincial government bodies. Overarching management principles for the portfolio of priorities in each forest management unit were also developed.

Communities and companies, both state and private sector, have effects on biodiversity but have little power to participate actively in the planning process in Vietnam. Companies have to operate under the regulation and monitoring of government departments, so it was felt that their impacts could be incorporated through their representative regulatory departments. The impact and requirements of communities, predominantly in plan implementation rather than in development, were incorporated into the process through the development of pilot communitybased natural resource management mechanisms, and lessons from these were fed into the process.

Conservation targets were identified by stakeholders, with technical support from WWF, and finalized through discussions with other provincial government departments. The targets were chosen using a table of attributes, based on those presented by Noss (1990) for landscape attributes and Pressey (2004) for planning functions, to ensure a full range of targets were captured. Targets were outlined in 2002 to enable focused data collection over the subsequent 3 years, with the goals being set in 2005 .

The systematic conservation planning software MARXAN (Ball \& Possingham, 200o) was used in combination with its ArcView (ESRI, Redlands, USA) interface, CLUZ (Smith, 2004), to identify the spatial priorities that met conservation targets. All of the targets had quantifiable goals. To determine landscape priorities the conservation targets were measured against the relative cost of managing a planning unit.

The relative cost of managing an area reflects the difficulty of removing the threats faced by the area. A model for the risk of forest clearance (Long, 2005) was used as a surrogate for the threat cost as this incorporated all significant factors that affect forest loss and may act as a suitable surrogate for natural resource harvest pressure. The forest-threat model also addresses landscape integrity issues such as edge effects, landscape fragmentation and risk of isolation.

Only 48 planning units were used and, as a result, simulated annealing in MARXAN produced few options, generally selecting the same portfolio every time, and thus a different approach was adopted. Provincial policy required the conservation of all forest land so the 48 planning units were prioritized and not selected to the exclusion of other areas. A four phase selection process was designed in collaboration with the Forest Protection Department based on each phase achieving different sub-targets towards the overall conservation target of conserving all forest land. The phases were: (1) all species goals met, $80 \%$ of pristine habitat included in priority 1 areas; (2) all species goals met, $80 \%$ of pristine habitat, $80 \%$ of watershed protection forest and $50 \%$ of all focal habitat representation included in priority 2 areas; (3) all species goals met, 90\% of remote forest and rivers, watershed protection forest and of all focal habitats included in priority 3 areas; (4) all goals met.

The final plan was integrated into the government planning system, resulting in the endorsement of the plan by the provincial government under the Quang Nam Biodiversity and Natural Resource Conservation Strategy 
2005-2020, which was endorsed by Quang Nam's Provincial People's Committee on 4 May 2005 (Decision 1332/ QD-UBND).

Terai Arc Landscape: planning for large vertebrate connectivity, with government planners

The selection of the Terai Arc Landscape for further planning resulted from several conservation priority-setting exercises. The landscape was selected as an important biodiversity conservation area representing the biodiversity of the Terai-Duar Savannah and Grasslands Ecoregion (Wikramanayake et al., 2001a) of the Himalayas. Several conservation targets in this landscape are large vertebrates, and the goal was an attempt to create a landscape sufficiently extensive to support metapopulations of tigers Panthera tigris, greater one-horned rhinoceros Rhinoceros unicornis and Asian elephants Elephas maximus along the Himalayan foothills, inner and outer valleys, and the Terai or floodplain grasslands. In 1997 a regional assessment of priority tiger conservation units identified eight landscapes along the length of the Himalayan foothills and alluvial grasslands of the Ganges and Brahmaputra rivers (Wikramanayake et al., 1999). A subsequent range-wide analysis of Asian elephant and rhinoceros populations identified the western Terai as harbouring globally important populations (Kemf \& Santiapillai, 2000).

The Terai-Duar Savannah and Grassland Ecoregion, globally significant for its biodiversity, is among the most converted and fragmented ecosystems in the Himalayan region (Wikramanayake et al., 2001b). The challenge was to conserve and restore habitat and maintain connectivity among core areas that harbour important populations of tigers, elephants and rhinos.

The primary focus of the planning was to connect core areas for the vertebrates and identify keystone habitat, especially the Saccharum spontaneum dominated riverine grasslands and riverine forests requiring restoration. The objectives were to conserve other threatened species, ensuring the livelihoods of the local people who still depend heavily on forest and natural resources, and protecting the ecological services, especially hydrological flows, that support biodiversity, local communities and local and national economies (E.D. Wikramanayake et al., unpubl. data).

The spatial extent of the landscape was between the Yamuna River in north-western India and Bhagmati River in Nepal, within which there are 11 protected areas. All the protected areas within this landscape harbour important tiger populations. The tiger's current range does not extend west of the Yamuna River and tigers have not been reported to the east of the Bhagmati River in recent years. The greater one-horned rhinoceros population in the western part of the Gangetic plain is also contained within these boundaries.
We set long-term (to 2020) conservation goals for the megaspecies: $(1)>500$ breeding tigers across the landscape, managed as a metapopulation with corridors between the protected areas facilitating dispersal; $(2)>1,500$ greater one-horned rhinoceros in three populations, centred around three protected areas complexes; (3) two ecologically and demographically viable Asian elephant metapopulations within their home ranges and migration pathways. Planning began with a reconnaissance survey to assess and ground-truth land and forest cover maps, and extensive discussions with local stakeholders, including villagers, local administrators, community-based organizations and non-governmental organizations, to obtain feedback regarding the feasibility and potential acceptance of a wildlife conservation landscape.

Tigers were selected as a focal species to identify potential corridors because, despite being a habitat generalist, they are less tolerant of disturbance and less likely to cross gaps than the other two megaspecies. Forest/land-cover maps, and tiger ecology and behaviour-related parameters, were then used in a GIS-based cost-distance model to identify potential corridors and bottlenecks that would need to be restored to improve connectivity between core areas (Wikramanayake et al., 2004).

Implementation faced challenges related to complications with stakeholders but progress has been made. Habitat restoration activities over the past 5 years in the Nepal Terai Arc Landscape has resulted in five corridors currently being used by tigers and three by rhinos and elephants.

\section{Discussion}

For the purposes of this article we evaluated WWF's landscape conservation planning efforts with respect to an operational model for implementing conservation action (Knight et al., 2006a,b). This model distinguishes between conservation assessment and conservation implementation, which when combined is considered conservation planning. This model emphasizes not only rigorous assessment of spatial priorities for action but the action itself, based on a perceived gap between assessment and action within the conservation community (Knight \& Cowling, 2003). The systematic rigour of conservation assessments has varied considerably within WWF but implementation has always been of paramount interest because of a traditionally low tolerance for planning (what we are actually calling 'assessment' here, sensu Knight et al., 2006a,b).

Table 1 illustrates how well the four case studies adhere to best practice as espoused by Knight et al. (2006a). The level of technical rigour varies but the relatively high level of implementation is consistent across all case studies. Here, we define implementation as the extent to which areas identified as priorities have been turned into 
TABLE 1 The degree to which the four WWF case studies contain Knight et al.'s (2006a) key ingredients for implementation of conservation planning.

\begin{tabular}{|c|c|c|c|c|}
\hline & Vogelkop & TransFly & Annamites & Terai \\
\hline \multicolumn{5}{|l|}{ Systematic assessment } \\
\hline Conduct a simple assessment even if data are limited & High & High & High & High \\
\hline Pursue goals of representation \& persistence & High & High & High & Low \\
\hline Integrate expert input \& systematic techniques & Medium & High & Medium & Medium \\
\hline Gather \& apply data useful to achieving goals & Medium & Medium & High & High \\
\hline Set quantified targets & Low & High & High & High \\
\hline Identification of stakeholders \& goals & Medium & High & High & High \\
\hline Assessments at different scales & Low & High & Low & Low \\
\hline Attention to assessment design & Low & High & Medium & Medium \\
\hline \multicolumn{5}{|l|}{ Assessment teams that include implementing organizations } \\
\hline Carefully recruit assessment teams & Medium & High & Medium & High \\
\hline Involve implementing organizations & High & High & Medium & High \\
\hline Involve the team in the planning-process design & Low & Medium & Low & Medium \\
\hline \multicolumn{5}{|l|}{ Focused collaboration to address stakeholders' needs } \\
\hline Identify key stakeholders first & Medium & High & High & High \\
\hline Design a collaboration programme with clear objectives & Low & Medium & Medium & Medium \\
\hline Avoid broad, unfocused stakeholder workshops & Medium & High & Medium & High \\
\hline Make the case for nature & Low & High & High & High \\
\hline \multicolumn{5}{|c|}{ Interpretation of assessment outputs \& mainstreaming of products } \\
\hline Deliver assessment outputs as useful planning products & Low & Medium & High & Medium \\
\hline Mainstream planning products into action & Medium & High & High & High \\
\hline
\end{tabular}

protected areas, are being managed explicitly in such a way as to preserve critical ecosystem function or are incorporated into government planning.

\section{Assessment}

The majority of WWF's spatial planning exercises until c. 2004 were carried out using the expert-driven process described earlier and in Mittermeier et al. (1994). This method is still used in some situations, depending on the discretion of the local programme staff. The method is relatively easy to organize provided that the relevant experts are available. A lead time of $2-3$ months is sufficient, although not optimal, to organize a large, c. 80 person, workshop that incorporates experts from the necessary range of biological disciplines. This method is relatively quick, requiring just 3-4 days once base maps have been prepared, and requires staff with only basic GIS skills. Expert and stakeholder support has been high, perhaps because experts and the government agency staff that respect them have confidence in the results (although this conclusion is based on our experience rather than any objective measure). The downsides of this method are that it is not as repeatable and is not often applied towards quantifiable goals, although the results themselves are quantifiable. Also, cost layers are not included, and the resulting portfolio is not the most efficient (in terms of using the minimum combination of cost and area).
Planning efforts focused at maintaining ecological processes, especially through the conservation of keystone species, also still occur, although these are usually the focus of programmes emphasizing species rather than the comprehensive conservation of a particular place.

There has been a perceptible shift in WWF towards more repeatable, systematic approaches (Margules \& Pressey, 2000). Since 2004 systematic conservation planning has become the rule rather than the exception because such analyses are more repeatable, emphasize quantifiable goals, are flexible to differing amounts of data, and can involve stakeholders and cost layers of various kinds (Naidoo et al., 2006; Pressey et al., 2007). Constraints include a requirement for a relatively high level of staff capacity (or consultants), which is not trivial for non-profit organizations, and possibly time, as consistent, region-wide datasets need to be collected and prepared.

\section{Implementation}

Implementation is a high priority concern. The four case studies have been implemented to varying degrees. The level of implementation is generally believed to be a function of a high level of expert, local and government support, which are in turn interrelated. The support was achieved through early and prolonged staff engagement of the various stakeholders, including their active participation in the assessment results. 
Costs (either threat costs or actual monetary costs) were considered in some of the case studies, and this probably increased the likelihood of implementation because, to the extent possible, priority areas were selected where costs were lower. Costs were not considered in the Vogelkop case study. The extent to which this will affect eventual implementation is unknown but is among an array of political and cultural factors that will affect the final outcome.

However, the completion of a conservation assessment will not necessarily result in the implementation of the plan. Although the ideal scenario is that WWF hands over a conservation assessment to government organizations to implement, more often WWF maintains ongoing engagement with relevant stakeholders to monitor the process of implementation (e.g. establishing protected areas and improving management). This process often takes years.

\section{A variety of approaches}

To summarize, WWF's initial efforts at large-scale planning were heavily skewed towards expert-driven workshops involving many experts and stakeholders but less repeatability (Mittermeier et al., 1994). More recently, many of WWF's ecoregion and landscape conservation planning efforts have involved more systematic approaches. These systematic conservation planning efforts have involved varying levels and formats of expert and stakeholder input.

This reflects a shift towards greater rigour as systematic conservation planning tools have become more prevalent, user-friendly and documented in the scientific literature. Most of WWF's ecoregion and landscape conservation planning now follows some variation of the Quang Nam and TransFly case studies.

The advantages of entertaining a variety of approaches are many. Each landscape has its own unique combination of culture, capacity, data, resources and geography. Providing for a variety of methods that can be chosen and mixed depending on the specific situation provides maximum flexibility to a diverse organization. In general, the variety of assessment approaches within WWF reflects differing time and budget constraints, the amount and quality of data available, perceived stakeholder capacity, WWF technical staff capacity, and the technical interest of WWF country programmes.

Pressey (2001) expressed concern that the large international non-governmental conservation organizations may form 'a kind of coalition with common planning methods.' The variety of approaches observed in this single region in one organization, WWF, indicates there may be little cause for concern.

There are downsides to this variety from an organizational perspective, however. There is little consistency among programmes in the organization and it is sometimes difficult to compare results. Theoretically a comparison would be possible. The focus would be on an objective evaluation of how much conservation was achieved, not only in terms of conservation management objectives (e.g. creation of protected areas) but including actual biodiversity outcomes (i.e. status of key habitats and species).

\section{More rigorous evaluation of methods desirable}

No systematic evaluation of conservation planning methods has been undertaken in WWF. Quantitative comparison of planning methods is difficult in general because exactly the same methods are rarely applied in different locations. It would be useful to compare systematic conservation planning efforts with expert-driven processes where the objectives are the same. There is one known example where The Nature Conservancy has applied both systematic and expert-only methods in the same location (the Gulf Coast of the USA; J. Morrison, pers. obs., 2009). There was significant overlap between the portfolios identified using expert-driven versus algorithm-driven methods. Although a rigorous analysis has not been performed, a comparison would be fair because the objectives of the two methods were similar (representation, persistence). One study comparing systematic conservation planning with expert-only methods (Cowling et al., 2003) was an unfair comparison, as the expert objectives (low-priority expansion of existing reserves) were different from the systematic methods (representation, persistence).

Future landscape-scale planning in WWF will probably incorporate increasingly sophisticated systematic conservation planning methods and explicitly factor in climate change impact and adaptation scenarios and monetary costs, which have been largely absent in WWF's landscape-scale planning to date. Expert-only methods may persist, driven by the perception, right or wrong, that they are less technically demanding, can be better understood by stakeholders, and that the results are similar to processes driven by planning software.

\section{Acknowledgements}

We wish to thank Thomas Barano for his contributions to the accounts regarding Indonesia, and Dr Robert Smith and Professor Nigel Leader-Williams for their contributions to the Vietnam account. We would also like to recognize the support and collaborations of all our local partners in each of the sites referenced. This paper is made possible by the generous support of the American people through the United States Agency for International Development (USAID). USAID's Global Conservation Program provided much support to WWF in the development of its landscape approach through a Leader with Associates Cooperative Agreement Award LAG-A-00-99-00048-00, and specific work for this paper was supported through Associate Cooperative Agreement Award EPP-A-00-04-00017-00. The contents of the paper 
are the responsibility of WWF, and do not necessarily reflect the views of USAID or the United States Government.

\section{References}

Ball, I.R. \& Possingham, H.P. (200o) MARXAN (v. 1.8.2): Marine Reserve Design using Spatially Explicit Annealing. A Manual Prepared for The Great Barrier Reef Marine Park Authority. Http://www.uq.edu.au/marxan/docs/marxan_manual_1_8_2.pdf [accessed 21 April 2009].

Baltzer, M.C., DAO, N.T. \& SHore, R. (eds) (2001) Towards a Vision for Biodiversity Conservation in the Forests of the Lower Mekong Ecoregion Complex-Technical Annex. WWF Indochina/WWF US, Hanoi, Vietnam and Washington, DC, USA.

Conservation Measures Partnership (2007) Open Standards for the Practice of Conservation. Http://conservationmeasures.org/CMP/ Site_Docs/CMP_Open_Standards_Version_2.o.pdf [accessed 21 November 2008].

Cowling, R., Pressey, R., Sims-Castley, R., le Roux, A., BaArd, E., Burgers, C. \& Palmer, G. (2003) The expert or the algorithm? Comparison of priority conservation areas in the Cape Floristic Region identified by park managers and reserve selection software. Biological Conservation, 112, 147-167.

Dinerstein, E., Olson, D., Atchely, J., Loucks, C., ContrerasBalderas, S., Abell, R. et al. (eds) (2000a) Ecoregion Based Conservation in the Chihuahuan Desert, A Biological Assessment and Biodiversity Vision. World Wildlife Fund/CONABIO/TNC/ PRONATURA/ITESM, Washington, DC, USA.

Dinerstein, E., Powell, G., Olson, D., Wikramanayake, E., A веLl, R., Loucks, C. et al. (200ob) A Workbook for Conducting Biological Assessments and Developing Biodiversity Visions for Ecoregion-Based Conservation. Conservation Science Program, WWF, Washington, DC, USA.

Kemf, E. \& Santiapillai, C. (2000) Asian Elephants in the Wild. WWF, Gland, Switzerland.

Knight, A.T. \& Cowling, R.M. (2003) Conserving South Africa's 'Lost' Biome: A Framework for Securing Effective Regional Conservation Planning in the Subtropical Thicket Biome. Report 44. Terrestrial Ecology Research Unit, University of Port Elizabeth, Port Elizabeth, South Africa.

Knight, A.T., Cowling, R.M. \& Campbell, B.M. (2006b) An operational model for implementing conservation action. Conservation Biology, 20, 408-419.

Knight, A.T., Driver, A., Cowling, R.M., Maze, K., Desmet, P.G., Lombard, A.T. et al. (2006a) Designing systematic conservation assessments that promote effective implementation: best practice from South Africa. Conservation Biology, 20, 739-750.

Kramer, P.A. \& Kramer, P.R. (2002) Ecoregional Conservation Planning for the Mesoamerican Caribbean Reef. WWF, Washington, DC, USA.

LONG, B. (2005) Identification of priority areas for integrated conservation management in Quang Nam province, Vietnam. $\mathrm{PhD}$ thesis, Durrell Institute of Conservation and Ecology, University of Kent, Canterbury, UK.

Margules, C.R. \& Pressey, R.L. (2000) Systematic conservation planning. Nature, 405, 242-253.

Mittermeier, R.A., Bowles, I.A., Cavalcanti, R.B., Olivieri, S. \& Da Fonseca, G.A.B. (1994) A Participatory Approach to Biodiversity Conservation: The Regional Priority Setting Workshop. Conservation International, Washington, DC, USA.

Naidoo, R., Balmford, A., Ferraro, P.J., Polasky, S., Ricketts, T.H. \& Rouget, M. (2006) Integrating economic costs into conservation planning. Trends in Ecology \& Evolution, 21, 681-687.

Noss, R.F. (1990) Indicators for monitoring biodiversity: a hierarchical approach. Conservation Biology, 4, 355-364.

Noss, R.F. (1992) The Wildlands Project: land conservation strategy. Wild Earth, Special Issue, 10-25.

Olson, D. \& Dinerstein, E. (1998) The Global 200: a representation approach to conserving the Earth's most biologically valuable ecoregions. Conservation Biology, 12, 502-515.

Olson, D.M., Dinerstein, E., Wikramanayake, E.D., Burgess, N.D., Powell, G.V.N., Underwood, E.C. et al. (2001) Terrestrial ecoregions of the world: a new map of life on Earth. BioScience, 51, 933-938.

Petocz, R.G. (1989) Conservation and Development in Irian Jaya: A Strategy for Rational Resource Utilization. E.J. Brill, Leiden, The Netherlands.

Pressey, R.L. (2001) Conservation planning-a young science playing in the big league. Society for Conservation Biology Newsletter, $8,2-3$.

Pressey, R.L. (2004) Conservation planning and biodiversity: assembling the best data for the job. Conservation Biology, 18, 1677-1681.

Pressey, R.L., Cabeza, M., Watts, M.E., Cowling, R.M. \& Wilson, K.A. (2007) Conservation planning in a changing world. Trends in Ecology \& Evolution, 22, 583-592.

Sмith, R.J. (2004) Conservation Land-Use Zoning (CLUZ) Software. Durrell Institute of Conservation and Ecology, Canterbury, UK. Http://www.mosaic-conservation.org/cluz [accessed 21 April 2009].

Wikramanayake, E.D., Carpenter, C., Strand, H. \& McKnight, M. (eds) (2001a) Ecoregion-Based Conservation in the Eastern Himalaya. Identifying Important Areas for Biodiversity Conservation. WWF and Center for Integrated Mountain Development, Washington, DC, USA.

Wikramanayake, E.D., Dinerstein, E., Loucks, C., Olson, D., Morrison, J., Lamoreux, J. et al. (2001b) Terrestrial Ecoregions of the Indo-Pacific: A Conservation Assessment. Island Press, Washington, DC, USA.

Wikramanayake, E.D., Dinerstein, E., Robinson, J.G., Karanth, U., Rabinowitz, A., Olson, D. et al. (1999) Where can tigers live in the future? A framework for identifying high priority areas for conservation. In Riding the Tiger: Conservation in a Human Dominated Landscape (eds J. Seidensticker, S. Christie \& P. Jackson), pp. 255-272. Cambridge University Press, Cambridge, UK.

Wikramanayake, E.D., McKnight, M., Dinerstein, E., Joshi, A., Guring, B. \& Smith, D. (2004) Designing a conservation landscape for tigers in human-dominated environments. Conservation Biology, 18, 839-844.

WWF (2005) WWF Standards of Conservation and Programme Management. Http://www.panda.org/what_we_do/how_we_work/ conservation/programme_standards/ [accessed 30 April 2009].

\section{Biographical sketches}

JOHN MORRISON has been providing spatial and strategic technical support to WWF field programmes for 11 years. Colby Loucks' research focuses on using spatial analyses to guide conservation planning ranging from global to local in scale. BARNEY LONG has 8 years' experience in conservation planning and implementation in Vietnam, where he ran WWF's conservation programme in Quang Nam province for 6 years. His main interest is species conservation at the site and landscape levels, incorporating protected area management and nature law enforcement. ERIC WIKRAMANAYAKE provides assistance to WWF's Himalayan ecoregion and species programmes. 